\title{
Modeling Separation Process for Sunflower Seed Mixture on Vibro-Pneumatic Separators
}

\author{
Igor SHEVCHENKO*, Elchyn ALIIEV*, Gintas VISELGA**, Jan Radek KAMINSKI*** \\ *Institute of Oilseed Crops of the National Academy of Agricultural Sciences, Institutska str. 1, Solnechnoye village, \\ Zaporozhye district, Zaporozhye region, 69093, Ukraine, E-mail: shevchenko9985@gmail.com; aliev@meta.ua \\ **Department of Mechanics and Materials engineering of Vilnius Gediminas Technical University, \\ J. Basanavicius str.28, 03224 Vilnius, Lithuania, E-mail: gintas.viselga@vgtu.lt \\ ***Department of Agricultural and Forest Machinery of Warsaw University of Life Sciences - SGGW, \\ Nowoursynowska str.159c,02-776 Warsaw, Poland,E-mail: jan_kaminski@sggw.pl
}

crossref http://dx.doi.org/10.5755/j02.mech.27647

\section{Introduction}

Task of finishing the seeding material of the parent components of sunflower hybrids and seeds of the primary links of seed production requires the use of more advanced technical methods. In order to obtain a homogeneous genetic seed material of the parent components, it is necessary to take into account all the traits in total, including the traits of the achene. Sunflower seeds vary widely in size, shape, and volume density [1-2]. For confectionery sunflower volume density of the achene is a quantitative trait that affects productivity of the plant.

Task of separating confectionery sunflower seeds by size is of great importance in calibration [3-4]. One of the ways to conduct this separation of sunflower seeds is to move them on vibrating screens to create a pseudo-fluidized layer [5]. Substantiation of processing and technological parameters of the specified process of seed calibration on sieves and development of the corresponding physical and mathematical model requires a solution.

As the analysis of sources and peer-reviewed scientific publications [6-7] has shown, many theories and methods of calculating the coordinate positions of seeds have been devoted to the study of the process of seed move- ment under the action of a vibrating sieve. In [8], a mathematical model of seed distribution on flat sieves was developed, that uses the theory of dimensional analysis, which rather superficially describes the technological process of separation. In research [9] the index of quality of technological process of seed division into fractions which is a criterion of efficiency, is introduced. Research [10] was also aimed at creating mathematical models that determine the influence of the main parameters of kinematics of the process on efficiency of seed separation. Research [11] was based on the physical and mathematical means of moving a material dot under the action of various forces, which does not take into account the interaction of particles with each other, that have a random initial position.

Aim of the research is to increase the efficiency of mechanical and technological distribution of sunflower seed mixture on adaptive vibro-pneumatic separators.

\section{Physical and mathematical means of seed movement}

Shape of the sunflower seeds can be approximated to the shape shown in Fig. 1. It consists of three spheres that intersect. The main characteristic of a seed is its effective diameter, which is defined as the diameter of a ball of equal volume.

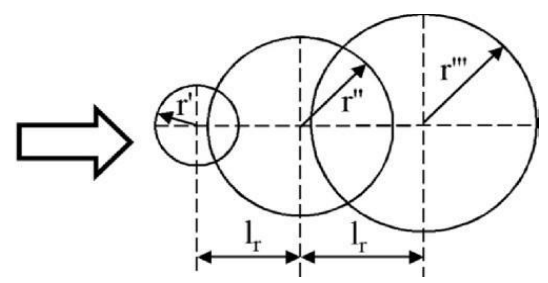

Fig. 1 Seed model

As the analysis of sources and peer-reviewed scientific publications [12-14] shows, many theories and methods of calculating the coordinate positions of seeds are devoted to the study of the process of seed movement (granular medium) on a vibrating surface under the action of vertical air flow. These studies are based on the physical and mathematical means of moving a material dot under the action of various forces, which does not take into account the interaction of seeds with each other, which have a random initial position. The problem of interaction of the seed flow with the air flow refers to the classical problem of gas dynamics of two-phase flows.
To study the process of moving sunflower seed mixture on a vibrating surface under the action of vertical air flow, it is necessary to determine the physical and mathematical means that will allow to obtain trajectories, force diagrams and sliding values during the movement of each seed in the mixture.

As a result of theoretical research, a system of differential equations of sunflower seed motions, as a granular gas, under the action of air flow (if necessary) and vibrating surface, taking into account the elastic-damping interaction, which allows to determine their position in space depending on physical and mechanical properties [15]: 


$$
\left\{\begin{array}{l}
\frac{d_{p} \overline{V_{p}}}{d t}=\bar{g}+\frac{\rho_{a}}{\rho_{p}} \bar{g}+\frac{\rho_{a}}{\rho_{p}} \frac{d_{a} \overline{V_{a}}}{d t}+\frac{\rho_{a}}{2 \rho_{p}} \frac{d}{d t}\left(\overline{V_{a}}-\overline{V_{p}}\right)+ \\
+\pi D_{p}^{2} \rho_{a} f_{M}(\operatorname{Re})\left(\overline{V_{a}}-\overline{V_{p}}\right)\left|\overline{V_{a}}-\overline{V_{p}}\right| /\left(2 \Omega_{p} \cdot \rho_{p}\right)+\overline{F_{\text {contact }}} /\left(\Omega_{p} \cdot \rho_{p}\right), \\
d_{p} \overline{S_{p}} / d t=\overline{V_{p}}, \quad d_{p} / d t=\partial / \partial t+\overline{V_{p}} \cdot \bar{\nabla}, \\
\overline{F_{\text {contact }}}=\left\{\begin{array}{ll}
\left(-K_{n} \overline{d_{n}}-N_{n} \overline{V_{n}}\right)+\left(-K_{t} \overline{d_{t}}-N_{t} \overline{V_{t}}\right), \quad \overline{S_{p A}}=\overline{S_{p B}} \\
0, & \overline{S_{p A}} \neq \overline{S_{p B}}
\end{array} .\right.
\end{array} .\right.
$$

where: $\Omega_{p}=\pi \cdot D_{p}^{3} / 6$ is seed volume, $\mathrm{m}^{3} ; D_{p}-$ effective seed diameter, $\mathrm{m} ; \rho_{p}$ - seed density, $\mathrm{kg} / \mathrm{m}^{3} ; \rho_{a}$ - air density, $\mathrm{kg} / \mathrm{m}^{3} ; \overline{V_{p}}-$ speed vector of seed movement, $\mathrm{m} / \mathrm{s} ; \overline{S_{p}}-$ seed movement vector, $\mathrm{m} ; \bar{g}$ - gravity acceleration, $\mathrm{m} / \mathrm{s}^{2}$; $\overline{V_{a}}$ - speed vector of air movement, $\mathrm{m} / \mathrm{s} ; t$ - time, $\mathrm{s}$; $f_{M}(R e)$ - ratio of viscous resistance; $R e$ - Reynolds number; $\overline{F_{\text {contact }}}$ - effort of interaction between seeds and surface, $\mathrm{N} ; K_{n}=4 E_{e q} \sqrt{d_{n} R_{e q}} / 3$ - normal ratio of rigidity of elastic component, $\mathrm{kg} / \mathrm{s}^{2} ; N_{n}=\sqrt{\left(5 K_{n} M_{e q}\right)} N_{n}$ damp - normal ratio of extinction of damping component, $\mathrm{kg} / \mathrm{s}$; $K_{t}=8 G_{e q} \sqrt{d_{t} R_{e q}}-$ tangential ratio of rigidity of elastic component, $\mathrm{kg} / \mathrm{s}^{2} ; N_{t}=\sqrt{\left(5 K_{t} M_{e q}\right)} N_{t \text { damp }}-$ tangential ratio of extinction of damping component, $\mathrm{kg} / \mathrm{s} ; \overline{V_{n}}, \overline{V_{t}}-$ normal and tangential components of relative velocity of seed surface in point of contact, $\mathrm{m} / \mathrm{s} ; N_{\text {damp }}$ - damping ratio; $R_{e q}=\left(2 / D_{A}+2 / D_{B}\right)^{-1}-$ equivalent radius of two seeds $\mathrm{A}$ and B, m; $E_{e q}=\left(\left(1-v_{A}^{2}\right) / E_{A}+\left(1-v_{B}^{2}\right) / E_{B}\right)^{-1}$ - equivalent Young modulus of two seeds $\mathrm{A}$ and $\mathrm{B}, \mathrm{Pa} ; M_{e q}=\left(M_{A}^{-1}+M_{B}^{-1}\right)^{-1}-$ equivalent mass of two seeds $\mathrm{A}$ and $\mathrm{B}, \mathrm{kg}$; $G_{e q}=\left(2 \cdot\left(2-v_{A}\right) \cdot\left(1+v_{A}\right) / E_{A}+2 \cdot\left(2-v_{B}\right) \cdot\left(1+v_{B}\right) / E_{B}\right)^{-1}-$ equivalent shear modulus of two seeds $\mathrm{A}$ and $\mathrm{B}, \mathrm{Pa} ; d_{n}, d_{t}-$ virtual overlapping of seeds $\mathrm{A}$ and $\mathrm{B}$ in normal and tangential directions, $\mathrm{m} ; M_{\mathrm{A}}, M_{B}-$ seed mass for seeds A and B (for surface $\left.M_{\text {wall }}=\infty\right), \mathrm{kg} ; D_{A}, D_{B}$ - effective diameters of seeds $\mathrm{A}$ and $\mathrm{B}$ (for surface $D_{\text {wall }}=\infty$ ), $\mathrm{m} ; E_{A}, E_{B}$ - Young modulus of seeds $\mathrm{A}$ and $\mathrm{B}, \mathrm{Pa} ; v_{A}, v_{B}$ - Poisson's ratio of seeds A and B.

Presented system of differential equations is the basis of the physical and mathematical means of numerical modeling of this process, which was implemented in the software package STAR-CCM +. The following were chosen as physical models for numerical simulations: $k-\varepsilon$ model of turbulence of a split flow, gravitational field, Van der Waals real gas model, model of discrete elements, model of multiphase interaction. To build physical and mathematical models, it was assumed that sunflower seeds are presented in the form of ellipsoids with a certain density and effective diameter.

\section{Method of numerical modeling}

To implement numerical modeling of the process of moving sunflower seeds under the action of a vibrating sieve and air flow, a calculation scheme was drawn up, which is used as basis of various seed cleaning and calibration machines (Fig. 2).

Previously, studies were conducted [3] to determine the following physical and mechanical, and morphological properties of sunflower seeds that are part of the mathematical model, namely: indicators that characterize the flowability of seeds (angle of natural slope $\varphi$ ); frictional properties of seeds (static coefficient of friction $f$ of sun-

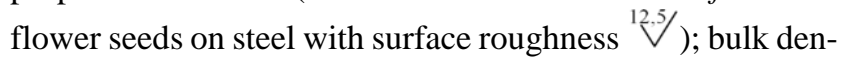
sity $\rho$; size and mass characteristics of seeds (length L, width $B$, thickness $T$, effective diameter $D_{p}$, weight of 1000 seeds $\left.m_{1000}\right)$.

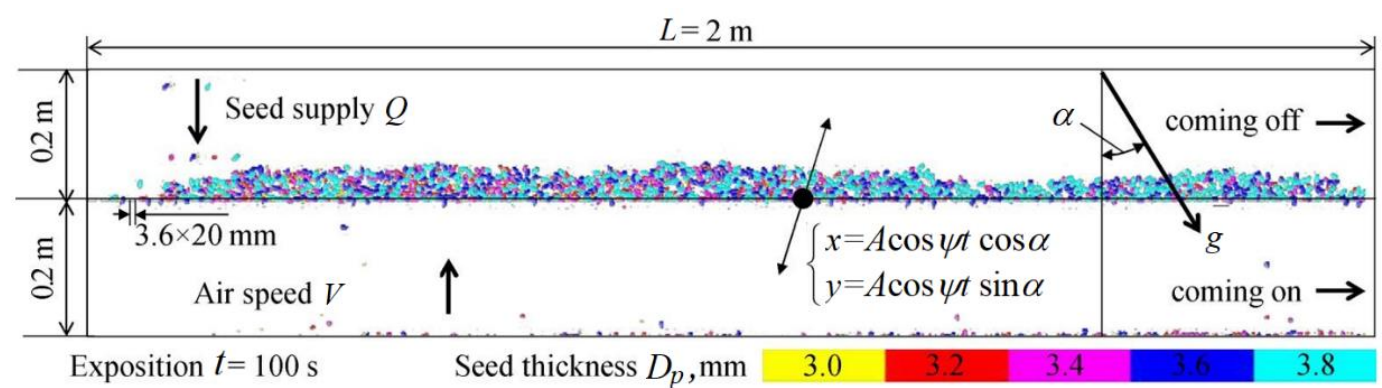

Fig. 2 Estimated scheme of the process of moving sunflower seeds under the action of a vibrating sieve and air flow

According to previous studies of the physical and mechanical properties of sunflower seeds of the Priority hybrid [16] for numerical modeling, the following average values were adopted: Poisson's ratio - 0.5; Young's modulus -
$0.2 \mathrm{MPa}$; density $-400 \mathrm{~kg} / \mathrm{m}^{3}$; skin friction ratio -0.8 ; normal recovery ratio -0.5 ; tangential recovery ratio -0.5 ; rolling resistance ratio -0.3 . The properties of the environment were as follows: environment - air; dynamic viscosity - 
$1,85508 \cdot 10^{-5} \mathrm{~Pa} \cdot \mathrm{s}$; Prandtl's turbulent number -0.9 ; gravity acceleration $-9.8 \mathrm{~m} / \mathrm{s}^{2}$; temperature $-293 \mathrm{~K}$; pressure $101325 \mathrm{~Pa}$. Size of the simulation grid cell was $0.001 \mathrm{~m}$.

According to the requirements set by breeding scientists, the most liquid fraction for sunflower seeds of the Priority hybrid is "fraction 3.6+" (sieve rise $3.6 \times 20 \mathrm{~mm}$ ). Therefore, further research was conducted based on this sieve. Relative useful area of this sieve was 0.56. Sieve performed periodic motion according to the law: $x=A \cos \psi t \cos \alpha, \quad y=A \cos \psi t \sin \alpha$, where $A$ is the amplitude of oscillations, $\mathrm{m} ; \psi$ - oscillation frequency, $\mathrm{s}^{-1}$. Seed mass flow was represented by 5 seed fractions of the same amount with different thickness $D_{p}$ in the range of 3.0-3.8 $\mathrm{mm}$ with a step of $0.2 \mathrm{~mm}$. Weight of 1000 seeds for determined effective diameter was in the range of 37.78-143.68 g (Table 1).

Table 1

Weight of 1000 seeds for determined effective diameter

\begin{tabular}{|l|c|c|c|c|c|}
\hline $\begin{array}{l}\text { Effective seed diameter } D_{p}, \\
\mathrm{~mm}\end{array}$ & 3,0 & 3,2 & 3,4 & 3,6 & 3,8 \\
\hline Weight of 1000 seeds $m_{1000}, \mathrm{~g}$ & 37,7 & 64,2 & 90,7 & 117,2 & 143,6 \\
\hline
\end{tabular}

The most important processing and technological parameters were taken as factors of numerical modeling: seed supply $Q(1100-2100 \mathrm{~kg} / \mathrm{h})$, sieve angle $\alpha\left(1-7^{\circ}\right)$, sieve frequency $\psi\left(4-6 \mathrm{~s}^{-1}\right)$, amplitude of sieve oscillations $A$ $(0.008-0.012 \mathrm{~m})$. Air flow rate was constant and was $V=3$ $\mathrm{m} / \mathrm{s}$. Angle of inclination of the sieve $\alpha$ was set by changing the angle of inclination of gravity acceleration $\bar{g}$, which is absolutely identical. Numerical simulations were performed on a full factorial experiment with a total number of experiments $-3^{4}=81$. The exposure started at $100 \mathrm{~s}$.

As a result of modeling, the concentration of each seed fraction that was reflected from the surface of the sieve (output) $\theta^{c}$ and passed through it (passage) $\theta^{n}$ was studied. Due to the fact that the size of the sieve hole was taken to be
$3.6 \times 20 \mathrm{~mm}$, a necessary condition for the qualitative separation of the seed mixture was to minimize the values of the concentrations of the fractions of $3.0 \mathrm{~mm}, 3.2 \mathrm{~mm}, 3.4 \mathrm{~mm}$, $3.6 \mathrm{~mm}$ in the output $\left(\theta_{D_{p}=3,0}^{c}, \theta_{D_{p}=3,2}^{c}, \theta_{D_{p}=3,4}^{c}, \theta_{D_{p}=3,6}^{c}\right.$ respectively). Therefore, as a research criterion, the total concentration of seeds in the output was taken, which is calculated by the following formula:

$$
\theta=\theta_{D_{p}=3,6}^{c}+\theta_{D_{p}=3,4}^{c}+\theta_{D_{p}=3,2}^{c}+\theta_{D_{p}=3,0}^{c} .
$$

Total productivity of the output and passage was taken as the quantitative criterion for assessing the effectiveness of the process of separation of sunflower seeds under the action of a vibrating sieve:

$$
q=q^{n}+q^{c}
$$

Also during the simulation, it was necessary to investigate the velocity vector field of the pseudo-fluidized layer of seeds on a vibrating screen.

\section{Results of numerical modeling}

As a result of modeling, a visualization of the technological process of moving sunflower seeds under the action of a vibrating sieve was obtained (Fig. 3). Histogram Fig. 3 illustrates the distribution of the seed concentration of the corresponding fraction in seed output $\theta^{c}$ and seed passage $\theta^{n}$, as well as the corresponding value of the total productivity $q$.

As a result of numerical simulation of the process of moving sunflower seeds under the action of a vibrating sieve, dependences of the change in total concentration $\theta$ and productivity $q$ on seed supply $Q$, sieve angle $\alpha$, sieve oscillation frequency $\psi$ and sieve amplitude $A$ were obtained (Figs. 4-5):

$$
\begin{aligned}
& q=-66637.82+162.22 \alpha+33577.7 A+0.013423 Q+2433.85 \psi-32.444 \alpha \psi+0.140125 Q \psi-227.453 \psi^{2} . \\
& \theta=198.853-1.733 \alpha-14055.2 A+284999 A^{2}+0.00852581 Q-39.653 \psi+0.303737 \alpha \psi+1364.8 A \psi- \\
& -0.00170516 Q \psi+2.27037 \psi^{2} .
\end{aligned}
$$

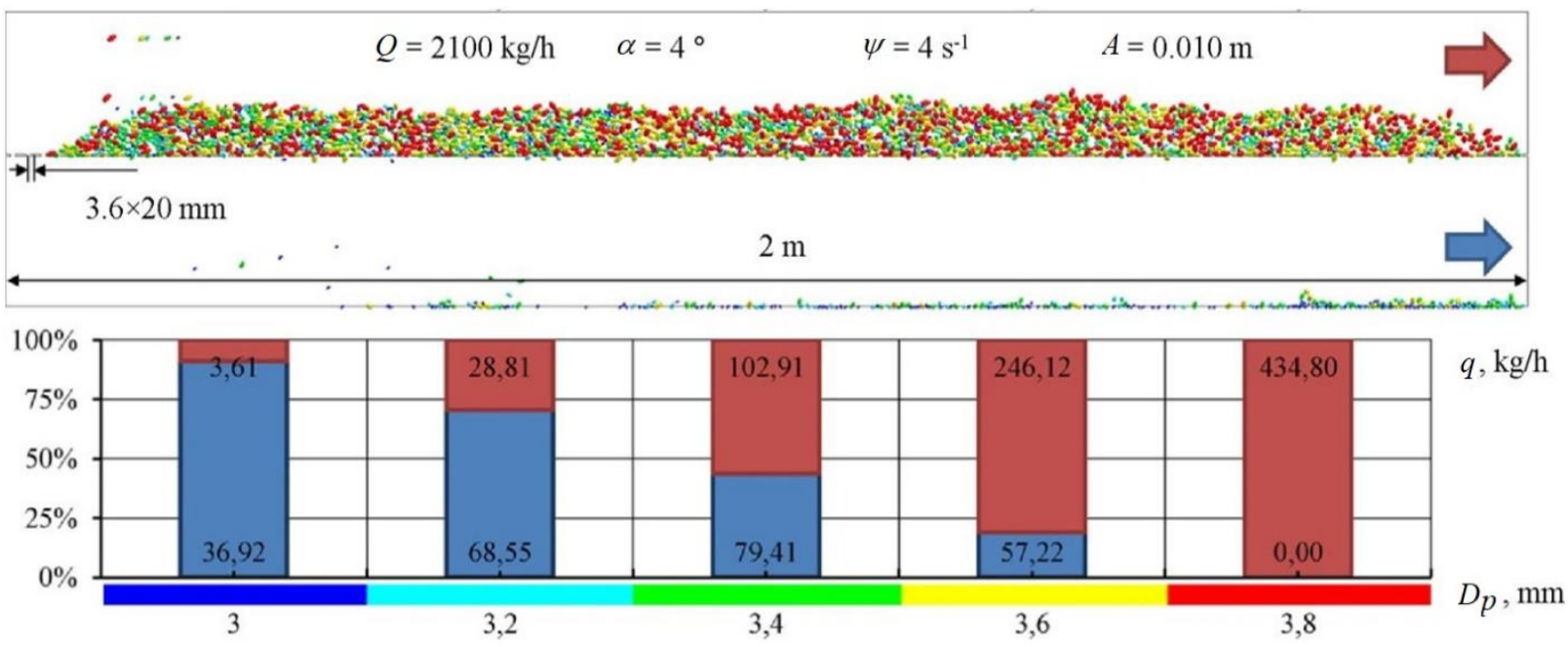

Fig. 3 Distribution of seed fractions at seed output and passage, formed under the action of a vibrating sieve 

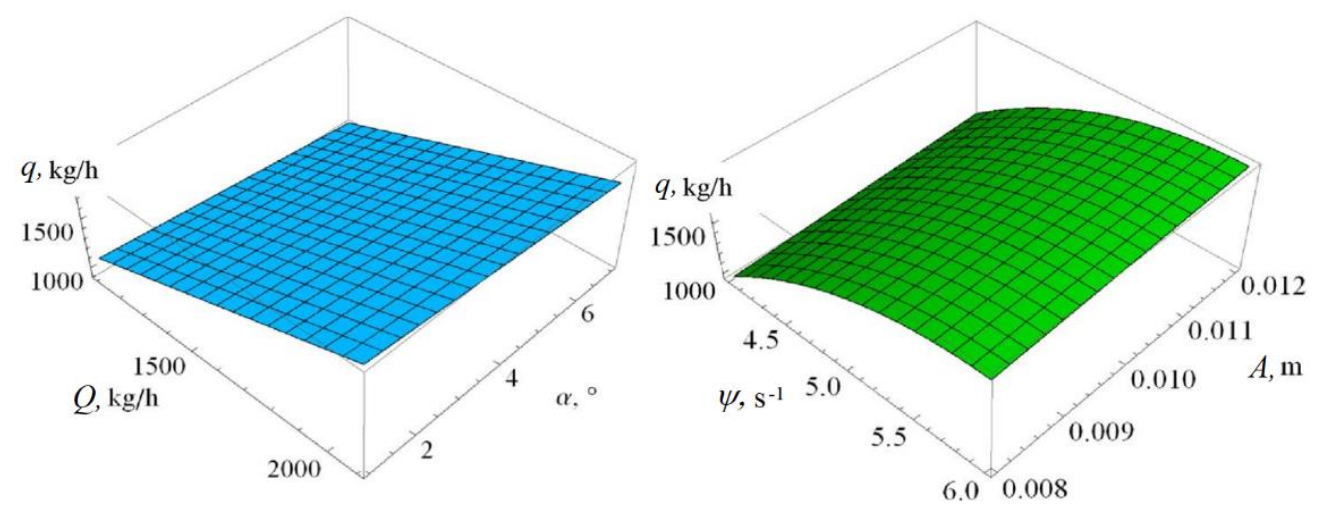

Fig. 4 Dependence of productivity $q$ on supply of seeds $Q$, angle of the sieve $\alpha$, sieve oscillation frequency $\psi$ and sieve amplitude $A$ at optimal parameters
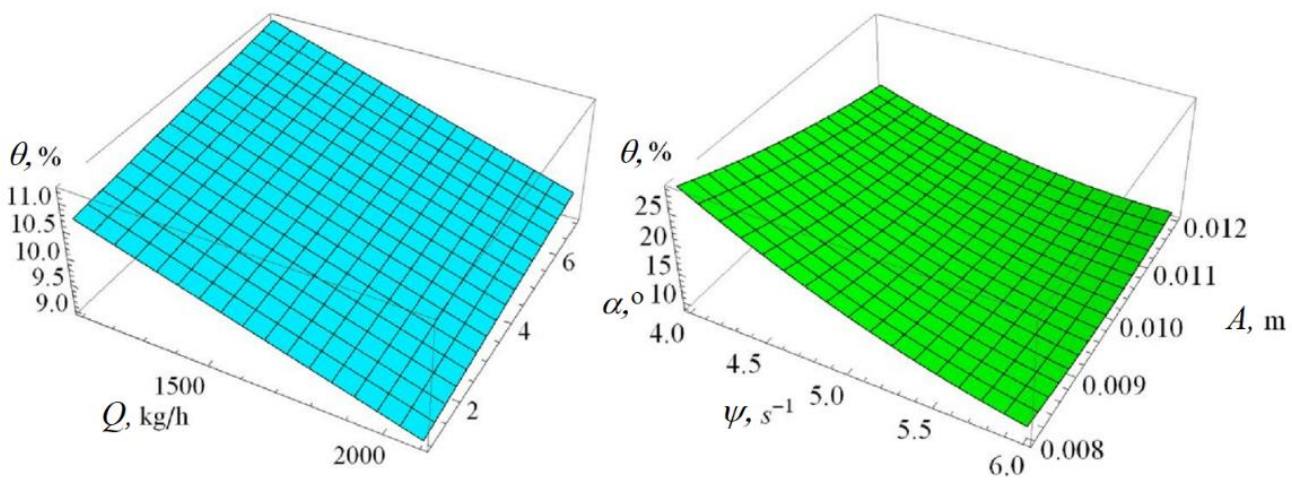

Fig. 5 Dependence of total seed concentration $\theta$ on seed supply $Q$, angle of the sieve $\alpha$, sieve oscillation frequency $\psi$ and sieve amplitude $A$ at optimal parameters

To provide efficiency of the seed separation process under the action of a vibrating sieve, it is necessary that its productivity $q$ be maximum and equal to the value of seed supply $Q=q=1202 \mathrm{~kg} / \mathrm{h}$, while the total seed concentration $\theta$ should be maximum $\theta=10.83 \%$, and $\alpha=1^{\circ}$, $\psi=5.62 \mathrm{~s}^{-1}, A=0.012 \mathrm{~m}$.

In addition to determining the rational processing parameters of separation of sunflower seeds under the action of a vibrating sieve, change in the velocity gradient of the pseudo-fluidized layer was investigated, according to which the seeds of the pseudo-fluidized layer perform translational and rotational spiral movement, changing their velocity direction vector $V$. In this movement there is a redistribution of particles of different sizes: smaller ones change downwards, and bigger ones - upwards. Due to this, the seeds are separated by size under the action of a vibrating sieve.

\section{Adaptive vibrating screen separator of sunflower seeds}

Design of the adaptive vibrating screen separator is presented in Fig. 6, and algorithm for implementing the technological process in Fig. 7 [17].

Separation process on the adaptive vibrating screen separator is as follows: the source grain or seed material enters the hopper 7 of the receiving device 6 . Then in the software of the personal computer 26 separation process starts (start=1). At the beginning point, valve 8 is installed in such a way as to ensure the maximum supply of grain or seed material $\left(q=q_{\max }\right)$. Two front and two rear airbags 2 and 3, respectively, are installed in such a way as to provide the maximum angle of the sieve frame $5\left(\alpha=\alpha_{\max }\right)$. Also at the beginning point, rotational frequencies of the rotors of the electric vibrators 12 are equal to the minimum value, accordingly, the vibration frequency of the sieve frame 5 is the largest $\left(\psi=\psi_{\min }\right)$. In addition to that, software of the personal computer 26 enters the values of the smallest size of the holes of sieve frame $5(q, \mathrm{~mm})$ and the required concentration of seeds for the output $\left(\Theta_{d}, \%\right)$, the size of which is greater than the smallest size of the holes of the sieve frame 5 . Change in the supply of grain or seed material $(q, \mathrm{~kg} / \mathrm{h})$ is as follows: if necessary, software of the personal computer 26 transmits a digital signal via electrical wires to the control unit of the stepper motor 23, where it is converted and fed to the stepper electric motor 9 , which sets the valve 8 in a certain position.

Change in the vibration frequency of the sieve frame $5(\psi, \mathrm{Hz})$ occurs in the following sequence: if necessary, software of the personal computer 26 transmits a digital signal via electrical wires to the motor control unit 24 . In the case of using an induction motor as electric vibrators 12 of, the control unit of the motors 24 changes the frequency of the power grind in the range from $0 \mathrm{~Hz}$ to $50 \mathrm{~Hz}$, which provides a change in the speed of its rotor. Otherwise, in the case of using DC motors as electric vibrators 12, the control unit of the motors 24 changes the power grid voltage in the range from $0 \mathrm{~V}$ to the nominal value, which changes the speed of its rotor. Rotation of the rotor of the electric vibrators 12 leads to a directly proportional change in the vibration frequency of the sieve frame 5. Changing the angle of the sieve frame $5\left(\alpha,{ }^{\circ}\right)$ is as follows: the personal computer 26 via electrical wires transmits the value of the angle in the form of a digital signal to the airbag control unit 25. Also, the airbag control unit 25 by electric wires constantly reads 
the pressure value from the front and rear electronic pressure sensors 15 and 18 , respectively. To maintain a constant pressure in the two front airbags 2 within 2.0-2.2 atm, airbag control unit 25 by means of electrical wires transmits a signal to the front high pressure electric valve 13 and the front atmospheric pressure electric valve 14 . After sending the signal to the front high pressure electric valve 13 , it opens and using pipelines, the two front airbags 2 are connected to the air receiver 19, while increasing the pressure in them. After sending the signal to the front atmospheric pressure electric valve 14 , it is opened and through the air filters 21 , the two front airbags 2 are connected to atmospheric pressure, while reducing the pressure in them. The pre-calibrated pressure value in the two rear airbags 3 corresponds to the value of the angle of the sieve frame 5. In the case of increasing (or decreasing) the angle of the sieve frame 5, the airbag control unit 25 by means of electrical wires transmits a signal to the rear high pressure electric valve 16 (or the rear atmospheric pressure electric valve 17). After sending the signal to the rear high pressure electric valve 16 , it opens and using pipelines, the two rear airbags 3 are connected to the air receiver 19 , while increasing the pressure in them. After sending the signal to the rear electric valve 17 , it opens and through the air filters 21 , the two rear airbags 3 are connected to the atmospheric pressure, while reducing the pressure in them. The compressor 20 when the pressure in the receiver 19 is turned on and injects air into itself, while increasing the pressure to a predetermined value.

Next, the grain or seed material falls on the plane of the sieve frame 5 of the basket 4 , where it is vibrated by oscillations of two electric vibrators 12 . While passing, grain or seed material on the plane of the sieve frame 5 is separated by the smallest geometric size into two fractions: 'output' and 'passage'. The 'passage' fraction includes seeds whose smallest geometric size is less than the value of the smallest size of the holes of the sieve frame 5. And the 'output' fraction can include seeds with different smallest geometric sizes. This is due to the fact that when moving the seeds in the plane of the sieve frame 5 , the probability of their passing depends on their orientation relative to the holes. 'Passge' fraction moves along the area of the passage through the sieve frame 5 and enters the unloading window for passage 11. And the 'output' fraction moves along the area of the passing through the sieve frame 5 and enters the unloading window for output 10 .

At the moment when 'output' fraction of grain or seed material is under the camera 22 , there is a process of photography with a frequency of no more than 10 seconds. Obtained images of the distribution of the 'output' fraction of grain or seed material in the area without holes of the sieve frame 5 are transmitted to a personal computer 26 . Next, in the personal computer 26, that has the appropriate software, obtained images are processed according to the developed algorithm.

Next, software of the personal computer 26, based on the entered values of the smallest size of the holes of the sieve frame $5(d, \mathrm{~mm})$, required concentration of seeds in the 'output' fraction $\left(\Theta_{d}, \%\right)$ and the obtained values of the concentration of the 'output' fraction of grain or seed mixture $\left(\theta_{d}, \%\right)$, performs the proposed algorithm and changes the input of feed of grain or seed material $(q, \mathrm{~kg} / \mathrm{h})$, the vibration frequency of the sieve frame $5(\psi, \mathrm{Hz})$ and the angle of inclination $\left(\alpha,{ }^{\circ}\right)$.

Use of the adaptive vibrating sieve separator allows to carry out technological processes of separation, and cleaning of grain and seed mixtures by the geometrical sizes with higher productivity, quality and the reduced complexity.

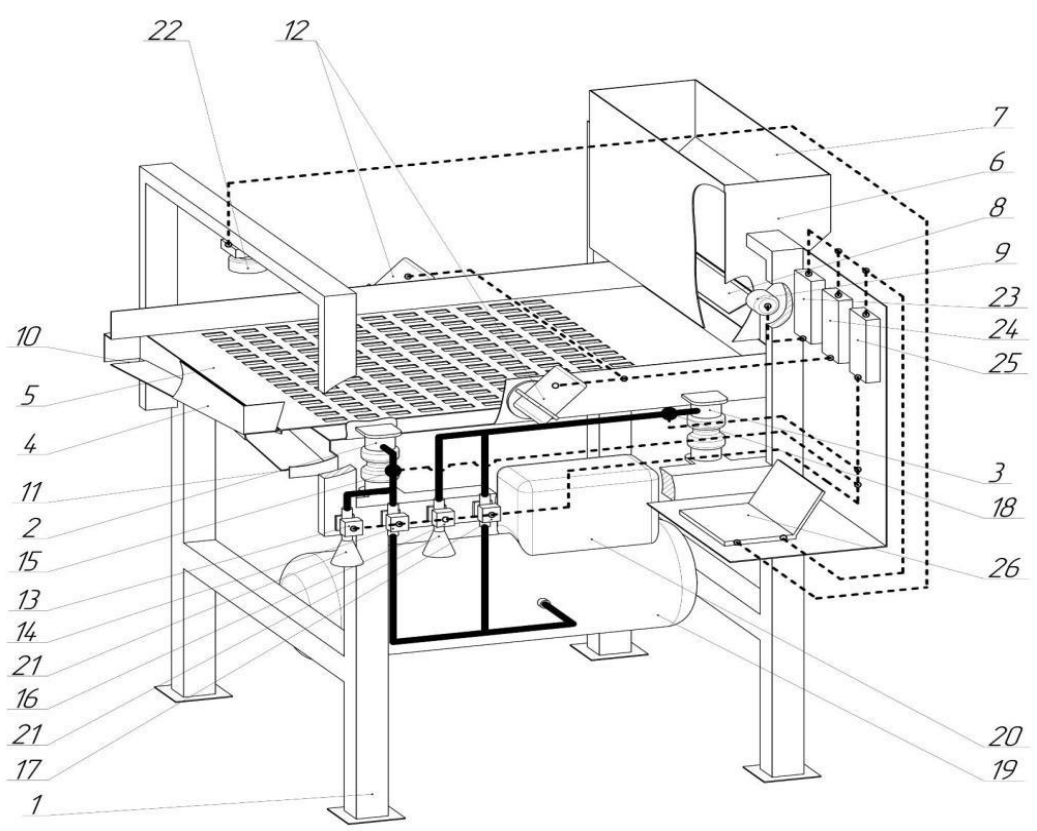

Fig. 6 Structural and technological scheme of adaptive vibrating screen separator: 1 - bed, 2 - front airbags, 3 - rear airbags, 4 - basket, 5 - sieve frame, 6 - receiving device, 7 - hopper , 8 - valve, 9 - rotor shaft of stepper electric motor, 10 - unloading window for output, 11 - unloading window for passage, 12 - electric vibrators, 13 - front high pressure electric valve, 14 - front atmospheric pressure electric valve, 15 - front electronic pressure sensor, 16 - rear high pressure electric valve, 17 - rear atmospheric pressure electric valve, 18 - rear electronic pressure sensor, 19 - air receiver, 20 - compressor, 21 - air filters, 22 - camera, 23 - stepper motor control unit, 24 - electric motor control unit, 25 - airbag control unit, 26 - personal computer 

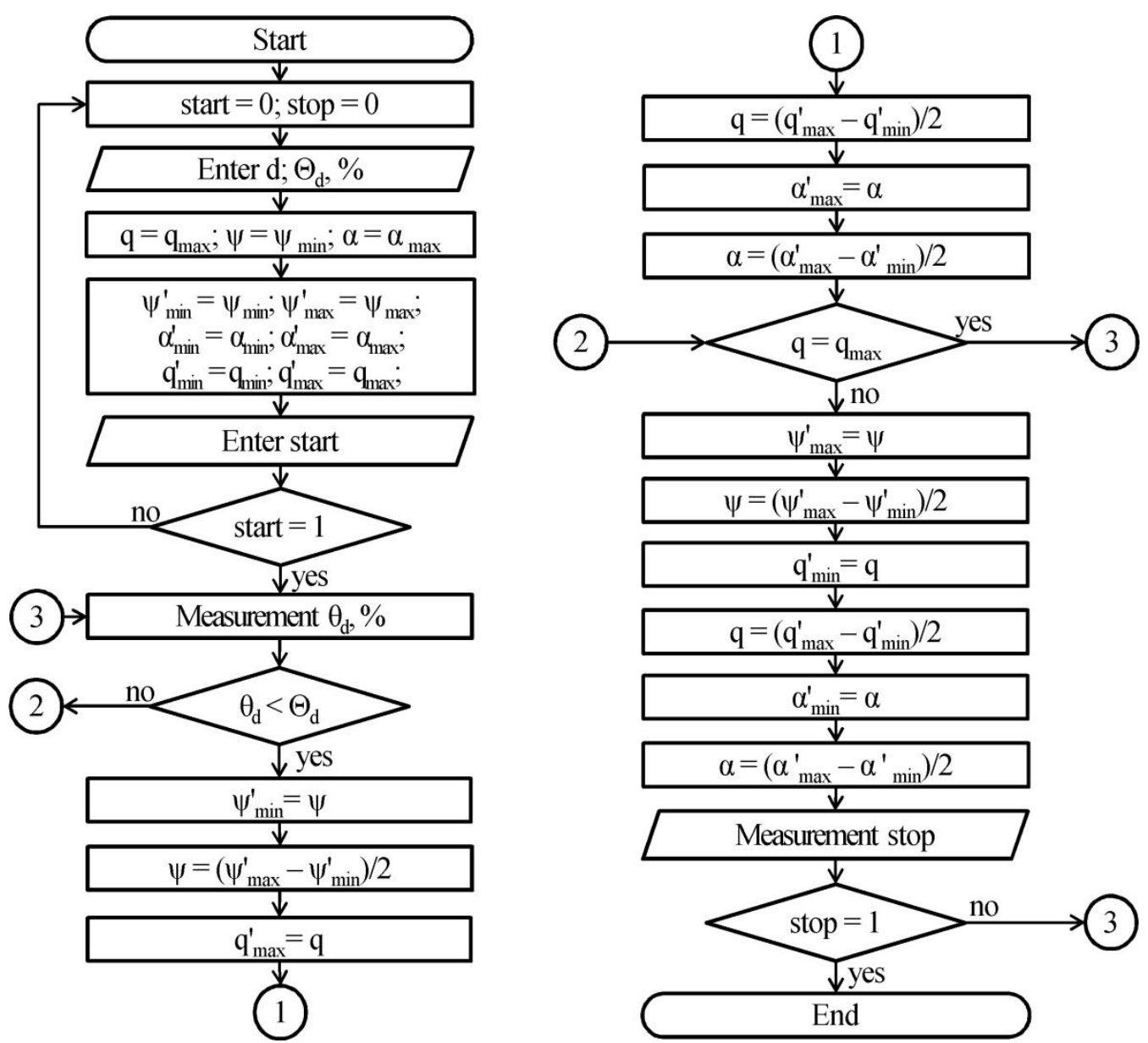

Fig. 7 Software algorithm: start - algorithm start variable; stop - algorithm stop variable; $d$ - value of the smallest size of cells of sieve frame 5, mm; $\Theta_{d}$ - required concentration of seeds in the output, $\% ; q$ - supply of grain or seed material, $\mathrm{kg} / \mathrm{h} ; \psi$ - vibration frequency of sieve frame $5, \mathrm{~Hz} ; \alpha$ - inclination angle of sieve frame $5,{ }^{\circ} ; \theta_{d}-$ measured total concentration of the fraction of grain or seed mixture, \%; «'»-denotes temporary variables; «min» - denotes minimum values of variable; «max»-denotes maximum values of variable

\section{Experimental verification of prototype equipment}

An ELP-USBFHD01M-BFV camera and a light source were mounted on the Cimbria Unigrain calibration machine for experimental verification of the structural and technological scheme of the adaptive vibrating screen separator. A general diagram of the operation of the experimental example of the adaptive vibrating screen separator is shown in Fig. 8.

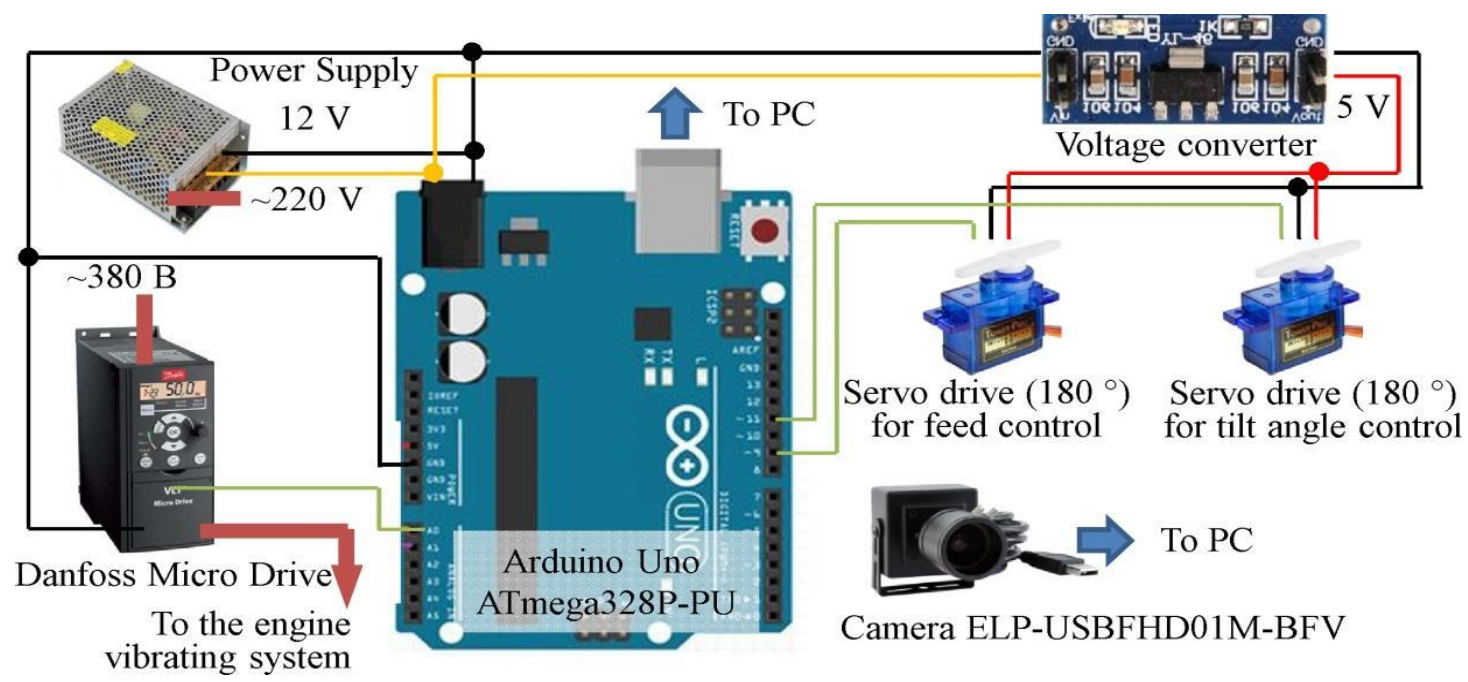

Fig. 8 Wiring diagram of the elements of the experimental example of the adaptive vibrating screen separator

The algorithm and implemented appropriate software based on Visual Studio $\mathrm{C}++$ and the OpenCV library analyzes all images obtained from the camera. As a result of the analysis, the seeds are automatically identified by taking 
successive points around the perimeter of the seeds and maximizing or minimizing the values in the following sequence: image upload; conversion into a 1-bit image (black seed on a white background); morphology analysis to remove noise and gaps; definition of contours, marking all the seeds in the image and calculating the length $L$, width $B$, area $S$ and the length of the perimeter $P$ of the seeds.

The image is processed in three stages (Fig. 9). First, the image of the selected rectangular area of the grain or seed fraction is converted from 24-bit (full color) to 1-bit (black and white) using the segmentation method to obtain black seeds on white [18]. This method is stable to the light level. Second, any noise or omission is removed. The software uses OpenCV Erode and Dilate functions based on morphological operations [19].

Third, using the Kenny Boundary Detector and the Huff transform, the OpenCV FindContour function [20] automatically determines the contour of each seed in a 1-bit image that acquires a set of coordinates (Fig. 10):

$$
P_{i}=\left(x_{i}, y_{i}\right)
$$

system $O(0,0)$ for all $P_{i}$ is defined as the upper left corner of the image. From the set of contour coordinates, the OpenCV ContourArea function calculates its area, and ArcLength [21] calculates the perimeter. Using these functions, the coordinates of the center of mass of the seeds are calculated as following:

$$
\left(x_{C}, y_{C}\right)=\left(\frac{1}{n} \sum_{i=0}^{n} x_{i}, \frac{1}{n} \sum_{i=0}^{n} y_{i}\right) .
$$

To measure the length $L$, the algorithm detects the maximum distance between points on the perimeter by calculating all the distances of the segment $h l_{i j}$ between all pairs of points of the contour (Fig. 10):

$$
h l_{i, j}=\sqrt{\left(x_{i}-x_{j}\right)^{2}+\left(y_{i}-y_{j}\right)^{2}} .
$$

The longest segment $L$ is determined by the follow-

The position of the starting point of the coordinate

$$
L=h l_{\max }=\max _{i, j}\left(h l_{i, j}\right), P_{h 0}\left(x_{h 0}, y_{h 0}\right), P_{h 1}\left(x_{h 1}, y_{h 1}\right) .
$$

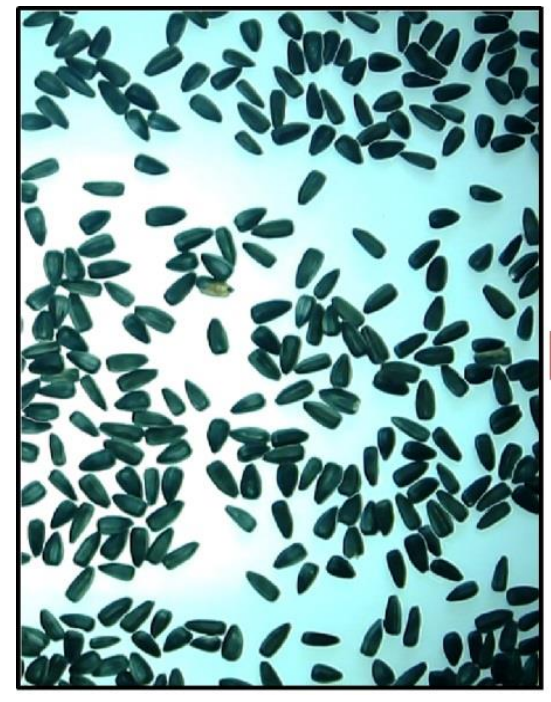

Original image of seeds

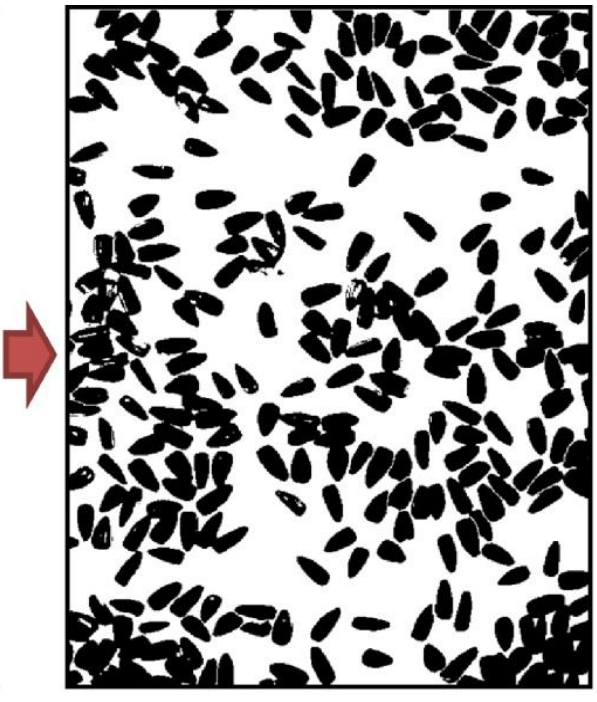

Transformed (black and white) image of seeds

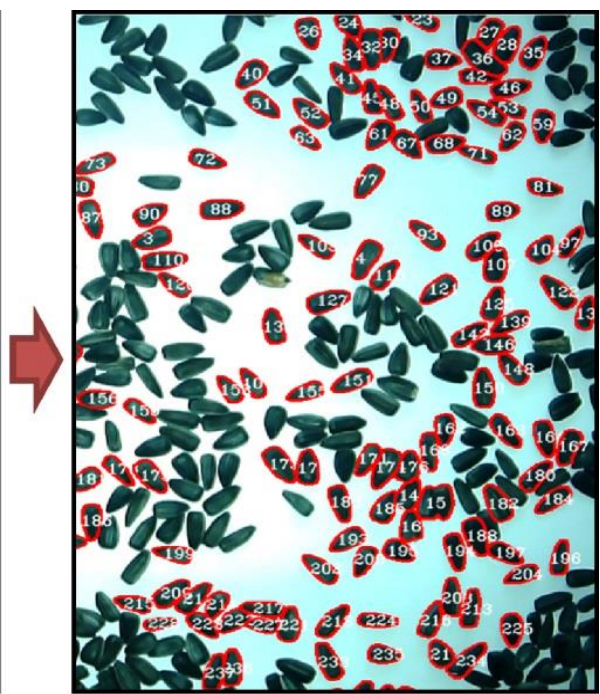

Seeds contours determination

Fig. 9 Image processing steps

To measure the width $B$ the algorithm detects the longest segment, which is perpendicular to the length $L$ (Fig. 10):

$$
\begin{aligned}
& w l_{i, j}=\sqrt{\left(x_{i}-x_{j}\right)^{2}+\left(y_{i}-y_{j}\right)^{2}}\left(\text { if } \frac{\left(y_{h 1}-y_{h 0}\right)}{\left(x_{h 1}-x_{h 0}\right)} \frac{\left(y_{i}-y_{j}\right)}{\left(x_{i}-x_{j}\right)}=-1\right) . \\
& B=w l_{\max }=\max _{i, j}\left(w l_{i, j}\right), P_{w 0}\left(x_{w 0}, y_{w 0}\right), P_{w 1}\left(x_{w 1}, y_{w 1}\right) .
\end{aligned}
$$

Next using the property of normal distribution, the procedure of stepwise selection of components included in the integrated curve of probability density distribution (Gaussian function):

$$
P(x)=A \exp \left[-(x-\bar{x})^{2} / 2 \delta^{2}\right]
$$

where: $P(x)$ is the probability density of the characteristic - $x$ (geometric size of the seed); $A$ is the amplitude of the Gaussian distribution; $x$ is the average value of the characteristic $\delta$ is the standard deviation of the characteristic $x$ from the average $\bar{x}$.

Using quadratic regression to the logarithm of the previous dependence:

$$
\ln P(x)=-\frac{x^{2}}{2 \delta^{2}}+\frac{\bar{x}}{\delta^{2}} x+\left[\ln A-\frac{\bar{x}^{2}}{2 \delta^{2}}\right]
$$


where the coefficient for the variable of the second order is $1 / 2 \delta^{2}$; with a variable of the first order $-\bar{x} / \delta^{2}$; free term $-\left(\ln A-\overline{x^{2}} / 2 \delta^{2}\right)$, all parameters of the normal distribution of the boundary section of the integral curve are determined. Sequential removal of selected Gaussians allows you to restore all components. Each component has its maximum which corresponds to the average value of the geometric size of the seed and the area normalized to one, which in percentage terms reflects the total concentration of a fraction of the grain or seed mixture $\left(\theta_{d}, \%\right)$.

Experimental test included two stages. First test was implemented with rational parameters of the vibrating screen separator. Second test was implemented by applying the automated control system of the process parameters of

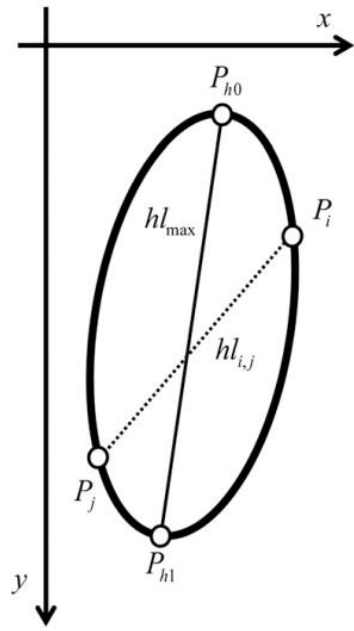

the vibrating screen separator according to the algorithm presented in Fig. 8. Each stage was carried out with 10 repetitions on seed mixtures that have different fractional composition of the Priority hybrid bred by the Institute of Oilseed Crops of NAAS. The criterion for assessing the quality of the process of separation of the seed mixture was the total seed concentration $\theta$ and productivity $q$.

Results of the experimental verification of the adaptive vibrating screen separator are presented in Table 2. Application of the developed algorithm for double separation of seed mixture on an adaptive vibrating screen separator allows to perform the technological process of separation of sunflower seed mixture by geometric dimensions with productivity $q=189 \pm 13 \mathrm{~kg} / \mathrm{h}$ and total seed concentration $\theta=3.4 \pm 1.3 \%$.

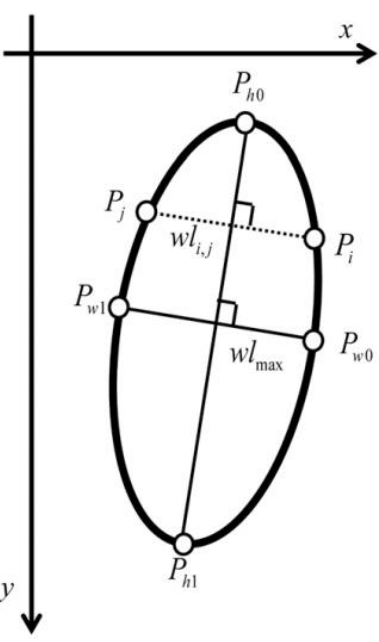

Fig. 10 Scheme for determining the parameters of the seeds

Table 2

Results of experimental verification of the adaptive vibrating screen separator

\begin{tabular}{|c|c|c|c|c|c|c|c|c|}
\hline \multirow{2}{*}{ Repition No } & \multicolumn{4}{|c|}{ First stage of separation } & \multicolumn{4}{c|}{ Second stage of separation } \\
\cline { 2 - 9 } & Rational parameters & Developed algorithm & \multicolumn{2}{c|}{ Rational parameters } & \multicolumn{2}{c|}{ Developed algorithm } \\
\cline { 2 - 9 } & $q, \mathrm{~kg} / \mathrm{h}$ & $\theta, \%$ & $q, \mathrm{~kg} / \mathrm{h}$ & $\theta, \%$ & $q, \mathrm{~kg} / \mathrm{h}$ & $\theta, \%$ & $q, \mathrm{~kg} / \mathrm{h}$ & $\theta, \%$ \\
\hline 1 & 330 & 9,1 & 375 & 7,3 & 178 & 8,0 & 180 & 2,9 \\
\hline 2 & 340 & 9,5 & 375 & 7,3 & 177 & 7,9 & 184 & 2,9 \\
\hline 3 & 348 & 9,9 & 364 & 6,7 & 183 & 7,2 & 185 & 2,3 \\
\hline 4 & 332 & 9,1 & 368 & 6,9 & 182 & 7,5 & 180 & 2,5 \\
\hline 5 & 391 & 12,0 & 422 & 9,5 & 156 & 10,1 & 209 & 5,1 \\
\hline 6 & 378 & 11,4 & 427 & 9,8 & 152 & 10,3 & 200 & 5,4 \\
\hline 7 & 326 & 8,8 & 375 & 7,3 & 178 & 8,0 & 178 & 2,9 \\
\hline 8 & 331 & 9,1 & 372 & 7,1 & 180 & 7,7 & 180 & 2,7 \\
\hline 9 & 395 & 12,2 & 416 & 9,3 & 159 & 9,9 & 211 & 4,9 \\
\hline 10 & 334 & 9,2 & 360 & 6,5 & 187 & 7,0 & 180 & 2,1 \\
\hline Average & 351 & 10,0 & 385 & 7,8 & 173 & 8,4 & 189 & 3,4 \\
\hline Standard deviation & 27 & 1,3 & 26 & 1,3 & 13 & 1,3 & 13 & 1,3 \\
\hline
\end{tabular}

Seed material purification in order to ensure high quality of the resulting product is an expensive process. These costs are associated with the constant reconfiguration of the equipment operating modes, which leads to productivity decrease due to prolonged downtime. In addition, when reconfiguring the equipment, there is a human subjective factor that reduces the quality of the separation process. The use of adaptive mechatronic systems makes it possible to eliminate equipment downtime and reduce the influence of the subjective factor, and this, in turn, increases equipment productivity and improves the quality of material separation.

\section{Conclusions}

As a result of research, dependences of the influence of moisture $W_{b}$ of sunflower seeds of the Institute of Oilseed Crops of NAAS on the effective diameter $D_{p}$, weight of 1000 seeds $m_{1000}$, bulk density $\rho$, natural slope angle $\varphi$, friction coefficient $f$ were established. As a result of theoretical research, a system of differential equations of motion of sunflower seeds as a granular gas under the action of air flow and vibrating surface was developed, taking into 
account the elastic-damping interaction, which allows to determine their position in space depending on physical and mechanical properties. Presented system of differential equations is the basis of the physical and mathematical means of numerical modeling of this process.

As a result of numerical simulation of the process of moving sunflower seeds under the action of a vibrating sieve, dependences of the change in total concentration $\theta$ and productivity $q$ on seed supply $Q$, sieve angle $\alpha$, sieve oscillation frequency $\psi$ and sieve amplitude amplitude $A$ were obtained. Provided that the efficiency of the seed separation process is adequate under the action of a vibrating sieve, it is necessary that its productivity $q$ be maximum and equal to the value of seed supply $Q=q=1202 \mathrm{~kg} / \mathrm{h}$, while the total seed concentration $\theta$ should be maximum $\theta=10.83$ $\%$, and $\alpha=1^{\circ}, \psi=5.62 \mathrm{~s}^{-1}, A=0.012 \mathrm{~m}$.

As a result of research, appropriate software has been developed to determine the fractional composition of seeds by photographic image, which is based on its conversion from 24-bit (full color) to 1-bit (black and white) using the method of segmentation, processing based on morphological operations and using detector for Kenny boundaries and Huff transformations to automatically determine the contour of each seed in a 1-bit image, followed by calculating the length $L$, width $B$, area $\mathrm{S}$, and length of the perimeter $P$ of the seed.

Based on the obtained theoretical and experimental dependences, an adaptive vibrating sieve separator with rationally agreed process parameters (seed supply $Q$, tilt angle $\alpha$ and oscillation frequency $\psi$ of the sieve) was developed thanks to the use of software based on the developed algorithm, which allows performing seed separation process by geometric dimensions with higher productivity $(q=189 \pm 13 \mathrm{~kg} / \mathrm{h})$ and quality (total seed concentration $\theta=3.4 \pm 1.3 \%)$.

\section{References}

1. Kirichenko, V. V. 2005. Sunflower breeding and seed production, Heliantus annus. 387p. (in Russian).

2. Vedmedeva, K. V.; Soroka, A. I. 2015. Influence of some mutant genes on certain agronomically important traits in sunflower, Helia 2: 21-22.

3. Ionescu, M.; Voicu, Gh.; Biriş, S.; Ştefan, E. M.; Ungureanu, N.; Dincă, M. N. 2016. Determination of some mechanical properties for oilseeds using uniaxial compression tests, INMATEH - Agricultural Engineering 49(2): 71-76.

4. Jafari, S.; Khazaei, J.; Arabhosseini, A.; Massah, J.; Khoshtaghaza, M. H. 2011. Study on mechanical properties of sunflower seeds, Electronic Journal of Polish Agricultural Universities 14(1): 1-11.

5. Schiehlen, W.; Eberhard, P. 2004. Technische Dynamik (in German). Stuttgart: Teubner. 324 p.

6. Alkhaldi, H.; Eberhard, P. 2006. Computation of screening phenomena in a vertical tumbling cylinder, Proceedings in Applied Mathematics and Mechanics (PAMM) Berlin, Germany, p. 83-84. http://dx.doi.org/10.1002/pamm.200610022.

7. Alkhaldi, H.; Eberhard, P. 2007. Particle screening phenomena in an oblique multi-level tumbling reservoir: A numerical study using discrete element simulation, Granular Matter 9: 415-429. http://dx.doi.org/10.1007/s10035-007-0042-6.

8. Casandroiu, T.; Popescu, M.; Voicu, G. 2009. A developing a mathematical model for simulating the seeds separation process on the plane sieves, U.P.B. Sci. Bull Series D 71(3): 17-27.

9. Bracacescu, C.; Gageanu, I.; Popescu, S.; Selvi, K.C. 2016. Researches concerning impurities separation process from mass of cereal seeds using vibrating sieves in air flow currents, Engineering for Rural Development 25-27.05: 364-370.

10. Sarsunov, V. A.; Kruglenja, V. E.; Kaminski, E.; Kubon, M. 2014. Flax seed separation with vibrating screens, Agricultural Engineering 3/151: 187-201. http://dx.medra.org/10.14654/ir.2014.151.071.

11. Broas, P. 2001. Advantages and problems of CAVEvisualisation for design purposes, Trans. VTT Symposium Virtual prototyping. Espoo, Finland, February 1 st: 73-81.

12. Delaney, G. W.; Cleary, P. W.; Hilden, M.; Morrison R. D. 2009. Validation of dem predictions of granular flow and separation efficiency for a horizontal laboratory scale wire mesh screen, Seventh International Conference on CFD in the Minerals and Process Industries CSIRO. Melbourne, Australia. 9-11 December 2009: 16.

13. Herrmann, H. J. 1993. Molecular dynamics simulations of granular materials, International Journal of Modern Physics C. 4(2): 309-316.

14. Ferrara, G.; Preti, U.; Schena, G. D. 1987. Computeraided use of a screening process model, APCOM 87, Proceeding of the Twentieth International Symposium on the Application of Computers and Mathematics in the Mineral Industries 2: 153-166.

15. Aliiev, E. B. 2017. Physical and mathematical apparatus of elastic-damping interaction of seeds under the action of a vibrating sieve. National interdepartmental scientific and technical collection, Design, manufacture and operation of agricultural machinery 47(part I.) Kropyvnytsky: CNTU. 31-39. (in Ukrainian).

16. Vedmedeva, K. V.; Machova, T. V.; Kirpitcova, N. M. 2017. The result of selection for large-fruitedness in the line and variety of sunflower. Collection of scientific works of the selection and genetic institute of the National Center for Seed Science and Variety Research 29 (69): 26-34. (in Ukrainian).

17. Aliev, E. B. 2019. Adaptive vibrating screen separator. Ukrainian patent for invention. 120235. MPK (2006) B07B 1/00, B07B 1/40 (2006.01), B07B 1/42 (2006.01), G05B 13/00, G05B 15/00. No a201811084. (in Ukrainian).

18. Wang, S., Wu, K., Yuan, Q., Liu, X., Liu, Z., Lin, X., Zeng, R., Zhu, H., Dong, G., Qian, Q. 2012. Control of grain size, shape and quality by OsSPL16 in rice. Nat Genet 44: 950-954.

19. OpenCV Developers Team. 2012. OpenCV Reference Manual. http://opencv.org/documentation.html (July 31, 2012).

20. Suzuki, S., Abe, K. 1985. Topological structural analysis of digital binary image by border following, Comput Vis Graph Image Process 30: 32-46.

21. Tanabata, T., Yamada, T., Shimizu, Y., Shinozaki, Y., Kanekatsu, M., Takano, M. 2010. Development of 
automatic segmentation software for efficient measurement of area on the digital images of plant organs, Hort Res 9: 501-506.

I. Shevchenko, E. Aliiev, G. Viselga, J. R. Kaminski

\section{MODELING SEPARATION PROCESS FOR SUNFLOWER SEED MIXTURE ON VIBRO- PNEUMATIC SEPARATORS}

S u m m a r y

Aim of the research is to increase the efficiency of the mechanical and technological process of separation of sunflower seed mixture on vibro-pneumatic separators, the principle of which is based on the interaction of seed flow with the surface having fluctuation-type vibration load by substantiating their efficient processing and technological parameters. A system of differential equations of sunflower seeds motions, as a granular gas, under the action of a vibrating surface was developed, taking into account the elastic-damping interaction and physical and mechanical properties of seeds. The presented system of differential equations is the basis of the physical and mathematical means of numerical modeling of this process, which was implemented in the software package STAR-CCM +. To build physical and mathematical models, it was assumed that sunflower seeds are presented in the form of ellipsoids with a certain density and effective diameter. As a result of numerical simulation of the process of moving sunflower seeds under the action of a vibrating sieve, dependences of the change in total concentration $\theta$ and productivity $q$ on seed supply $Q$, sieve angle $\alpha$, sieve frequency $\psi$ and sieve amplitude $A$ were obtained. As a result of numerical simulation of the process of moving sunflower seeds under the action of a vibrating surface, dependences of the change of filling factor $\chi$, distribution coefficient $\delta$ and productivity $q$ on seed supply $Q$, vibration surface angles $\alpha$ and $\beta$, oscillation frequency $\psi$, oscillation amplitude $A$ and set air velocity $V$ were obtained. Theoretical provisions were implemented and tested in the development of an adaptive vibrating screen separator of sunflower seeds (Ukrainian patent No 120235).

Keywords: seeds, separation, vibro-pneumatic separator, modeling, STAR-CCM+, parameters, optimization.

Received December 07, 2020 Accepted August 07, 2021 